\section{Pitfalls in the management of TB-associated uveitis}

S Basu and T Das

treated with periocular and topical steroids. Anti-tubercular therapy (ATT) was not given though his tuberculin reaction was $16 \times 16 \mathrm{~mm}$ after $48 \mathrm{~h}$, and other tests were negative. After 7 years, he developed an intra-cranial tuberculoma (Figure 1), for which ATT was initiated. After 5 months of ATT, he complained of reduced vision in the left eye. We detected anterior uveitis in the right eye and recurrent intermediate uveitis in the left eye, which we treated with oral and topical prednisolone while continuing ATT. Vitritis reduced significantly and BCVA improved to 20/60 in left eye after 3 months. The cerebral lesion also regressed completely.

\section{Case 2}

A 36-year-old woman, presenting with a solitary choroidal granuloma in the left eye (Figure 2a), was diagnosed as probable ocular sarcoidosis, based on raised serum angiotensin convertase enzyme (SACE) levels, and negative tuberculin test and chest radiography. The lesion regressed well with oral prednisolone (Figure 2b). After 3 months later, computerised tomography revealed intra-cranial spaceoccupying lesion in her left parietal lobe, diagnosed as intra-cranial tuberculoma (Figure 2c). Tuberculin reaction was now $15 \times 15 \mathrm{~mm}$. ATT was started. But 2 weeks later, she developed a chorio-retinitis patch in the left eye, adjacent to the scar of the previous lesion (Figure 2d). The lesion subsided with intravenous methyl prednisolone (IVMP, $1 \mathrm{mg} / \mathrm{kg}$ for 3 days) followed by oral prednisolone.

\section{Case 3}

A 45-year-old man with decreased vision in the left eye (BCVA: 20/80) for 1 month showed serpiginous choroiditis in the left eye (Figures $3 a$ and b). Tuberculin test was $23 \times 24 \mathrm{~mm}$, chest radiography was normal and vitreous biopsy was negative for mycobacterial DNA. We started ATT, adding oral prednisolone $(1 \mathrm{mg} / \mathrm{kg}$ body weight) after 2 days (Figure 3c). However,
Retina-Vitreous Services, LV Prasad Eye Institute, Bhubaneswar, India

Correspondence: S Basu, Retina-Vitreous Services, LV Prasad Eye Institute, Patia, Bhubaneswar - 751 024, Orissa, India Tel: + 916743989 202; Fax: +91674 3987130 . E-mail: basu@lvpei.org

Received: 9 March 2010 Accepted in revised form: 3 July 2010

Published online: 13 August 2010 
after 1 week, BCVA deteriorated to 20/160 in the left eye with clear advancement of choroiditis margins. IVMP ( $1 \mathrm{mg} / \mathrm{kg}$ for 3 days) followed by oral prednisolone led to resolution of lesions.

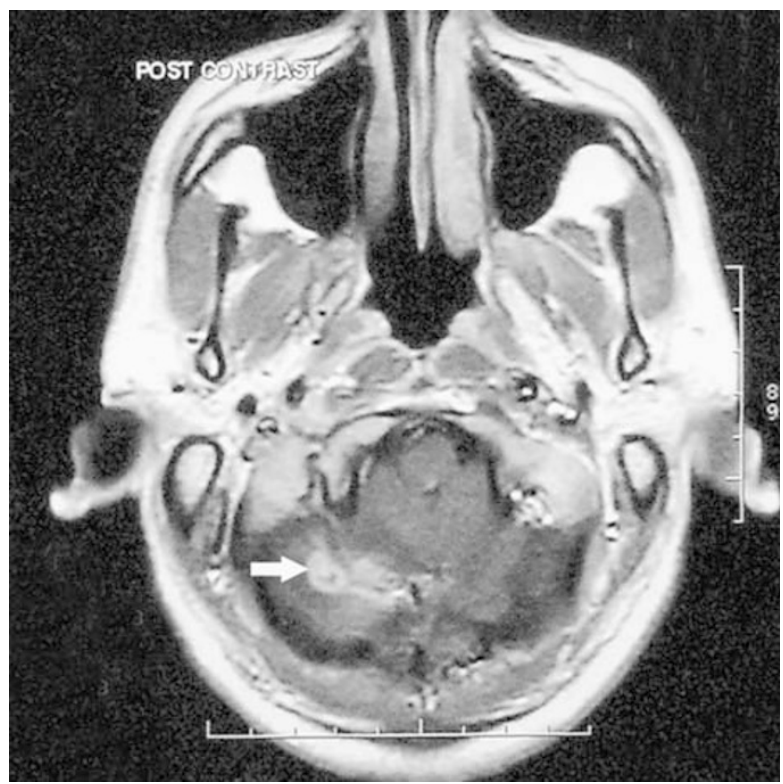

Figure 1 Magnetic resonance imaging of the brain with gadolinium enhancement (T1-weighted image) showing ringenhancing lesion (arrow) involving the right cerebellar hemisphere (patient 1).

\section{Case 4}

A 24-year-old man presented with extensive pigmentary scarring in the right eye and serpiginous-like choroiditis with focal skip lesions in the left eye (Figures $4 a$ and b). Tuberculin test was $20 \times 20 \mathrm{~mm}$ at $48 \mathrm{~h}$; chest radiograph was normal. We explained the need for starting ATT, but the patient refused fearing side effects of the medication. We treated with IVMP and oral prednisolone, which caused resolution of serpiginous lesions. However, 4 months later, reactivated lesions of serpiginous choroiditis were seen in the left eye (Figure 4c). Vitreous biopsy detected mycobacterial DNA. The patient agreed for ATT. Oral prednisolone ( $1 \mathrm{mg} / \mathrm{kg}$ body weight) was added after 2 days. But 1 week later, the choroiditis lesions were found enlarged and associated with serous retinal detachment (Figure 4d). IVMP therapy finally caused resolution of all lesions.

\section{Discussion}

These case reports illustrate several pitfalls that may occur in diagnosis and management of TB-associated uveitis. In cases 1 and 2, failure to treat tubercular uveititis with ATT (tuberculin reaction was positive in case 1 and false negative in case 2), along with unopposed steroid therapy, led to a serious adverse systemic consequence - intra-cranial tuberculoma. In case 4 , non-inclusion of ATT during initial therapy
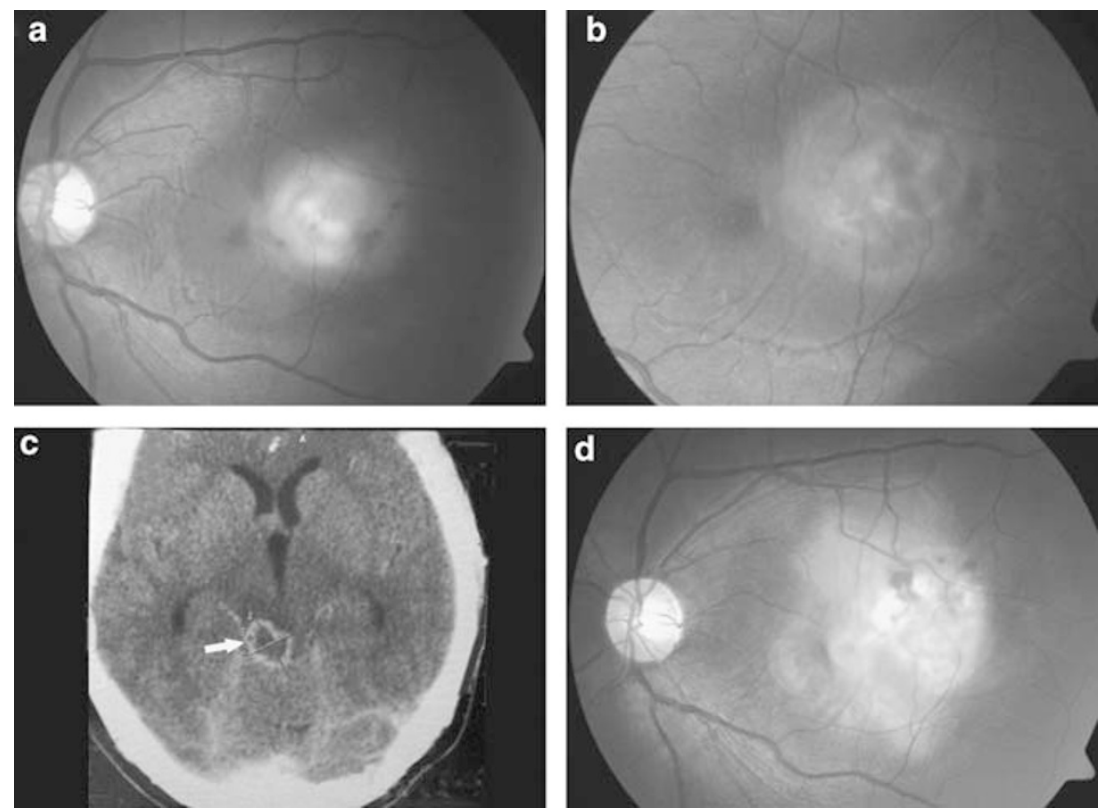

Figure 2 (a) Solitary choroidal granuloma temporal to fovea in the left eye of patient 2. (b) Resolution of lesion after treatment with oral prednisolone. (c) Computerised tomography scan of the head with contrast enhancement showing ring-enhancing lesion (arrow) on superior convexity of the left parietal lobe, suggestive of intra-cranial tuberculoma. (d) Large patch of chorio-retinitis at the site of the previous lesion, extending on to the fovea, after initiation of anti-tuberculosis therapy (ATT). 

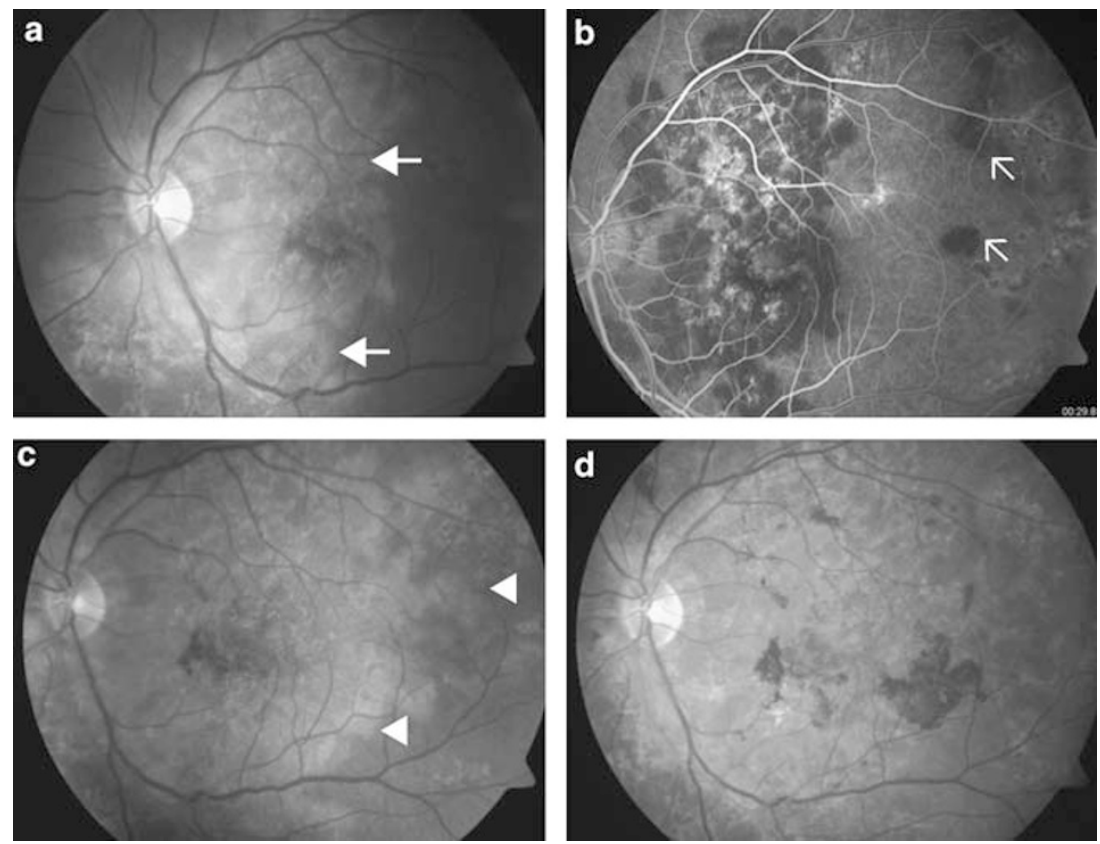

Figure 3 (a) Flat, confluent peripapillary lesion with central scarring and active pseudopodal edges (bold arrows) in the left eye of patient 3. (b) Fluorescein angiogram (early venous phase) of the same eye showing the extent of lesion, and presence of focal, skip lesions (arrows) temporally. (c) Progression of the lesion (arrow heads) after 1 week of ATT, well beyond the fovea (d) Superficial scarring seen after complete resolution of the lesion.
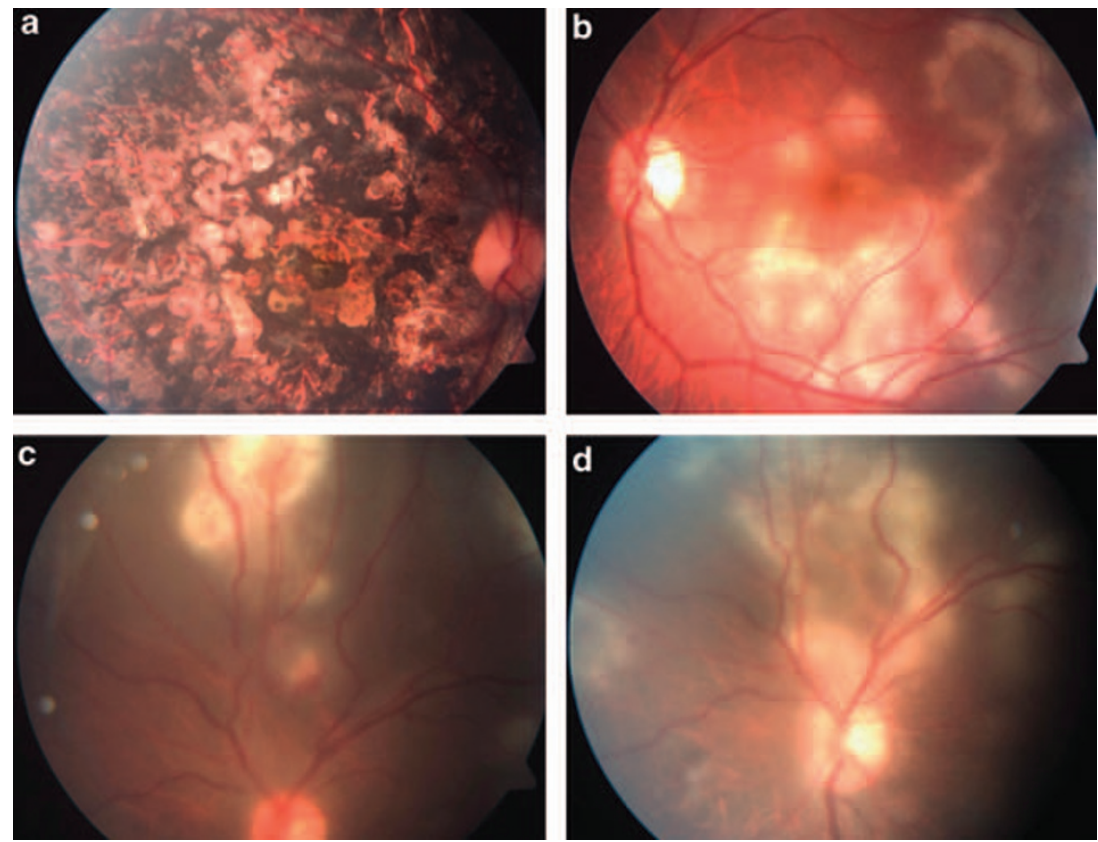

Figure 4 (a) Right fundus of patient 4 showing extensive scarring with heavy pigmentation at the posterior pole. (b) Left fundus of the same patient showing flat confluent lesion with active, pseudopodal edges, and focal, skip lesions. (c) Reactivated serpiginous lesions in the left eye of patient 4 after 4 months of anti-inflammatory therapy. (d) Increase in size of thosel lesions with surrounding serous retinal detachment, after introduction of ATT.

(in spite of strong evidence of latent TB) caused recurrent disease in the eye. False-negative tuberculin reaction could occur in poor nutrition and general health, overwhelming acute illness, immunosuppression, or early stages of infection. ${ }^{4}$ Interferon- $\gamma$-release assay could have been useful here. Also, as in case 2, SACE levels could be high in some forms of pulmonary TB. ${ }^{5}$ 
Table 1 Comparison of characteristics of patients with paradoxical reactions in the eye during anti-tuberculosis therapy

\begin{tabular}{|c|c|c|c|c|}
\hline & Case 1 & Case 2 & Case 3 & Case 4 \\
\hline Initial lesion & Intermediate uveitis & Choroidal granuloma & $\begin{array}{l}\text { Serpiginous-like } \\
\text { choroiditis }\end{array}$ & $\begin{array}{l}\text { Re-activated } \\
\text { serpiginous-like choroiditis }\end{array}$ \\
\hline Systemic disease & $\begin{array}{l}\text { Yes (intra-cranial } \\
\text { tuberculoma) }\end{array}$ & Yes (intra-cranial tuberculoma) & No & No \\
\hline Post-ATT lesion & $\begin{array}{l}\text { Intermediate uveitis } \\
\text { (recurrence) }\end{array}$ & $\begin{array}{l}\text { Choroidal granuloma } \\
\text { (recurrence from healed lesion) }\end{array}$ & $\begin{array}{l}\text { Progression of } \\
\text { existing lesion }\end{array}$ & $\begin{array}{l}\text { Enlargement of } \\
\text { existing lesion }\end{array}$ \\
\hline Onset time & 5 months & 2 weeks & 1 week & 1 week \\
\hline Concurrent steroids & None & None & Yes & Yes \\
\hline Outcome & Resolution & Resolution & Resolution & Resolution \\
\hline Follow-up & 20 months & 18 months & 17 months & 15 months \\
\hline
\end{tabular}

Abbreviation: ATT, anti-tuberculosis therapy.

Additionally, a common feature of all the four cases was the appearance of new lesions (cases 1 and 2) or worsening of existing lesions (cases 3 and 4) in the eye, after initiating ATT (Table 1). Such paradoxical reactions, also called Jarisch-Herxheimer reactions are an established phenomenon in patients with systemic $\mathrm{TB}^{6,7}$ and have also been described for ocular TB. ${ }^{2,8}$ Suggested mechanisms include release of mycobacterial antigens after ATT, strengthening of host's immune response, and decrease in suppressor mechanisms. ${ }^{6}$ In cases 3 and 4, however, it may also be argued that inadequate antiinflammatory therapy resulted in worsening of ocular lesions. Rifampicin has been shown to reduce the bio-availability of prednisolone by $66 \% .^{9}$ In all the four cases, these reactions resolved by adding/increasing corticosteroid therapy.

Tubercular uveitis therefore warrants a strong index of suspicion, and upon diagnosis, a combined anti-tubercular and anti-inflammatory therapy, for successful outcome.

\section{Summary}

What was known before

- Various manifestations of TB-associated uveitis.

- Treatment of TB-associated uveitis.

- Existence and nature of paradoxical reactions after ATT for systemic tuberculosis.

\section{What this study adds}

- Various pitfalls that may occur in the diagnosis and treatment of TB-associated uveitis.

- Spectrum of paradoxical reactions in the eye after ATT for intra- and extra-ocular tuberculosis.

\section{Conflict of interest}

The authors declare no conflict of interest.

\section{References}

1 Singh R, Gupta V, Gupta A. Pattern of uveitis in a referral eye clinic in north India. Indian J Ophthalmol 2004; 52: 121-125.

2 Gupta V, Gupta A, Rao NA. Intra-ocular tuberculosis - an update. Surv Ophthalmol 2007; 52: 561-586.

3 Vasconcelos-Santos DV, Zierhut M, Rao NA. Strengths and weaknesses of diagnostic tools for tuberculous uveitis. Ocul Immunol Inflamm 2009; 17: 351-355.

4 Holden M, Dubin MR, Diamond PH. Frequency of negative intermediate-strength tuberculin sensitivity in patients with active tuberculosis. $N$ Engl J Med 1971; 285: 1506-1509.

5 Brice EA, Friedlander W, Bateman ED, Kirsch RE. Serum angiotensin-converting enzyme activity, concentration, and specific activity in granulomatous interstitial lung disease, tuberculosis, and COPD. Chest 1995; 107: 706-710.

6 Afghani B, Lieberman JM. Paradoxical enlargement or development of intracranial tuberculomas during therapy: case report and review. Clin Infect Dis 1994; 19: 1092-1099.

7 Cheng SL, Wang HC, Yang PC. Paradoxical response during anti-tuberculosis treatment in HIV-negative patients with pulmonary tuberculosis. Int I Tuberc Lung Dis 2007; 11: 1290-1295.

8 Cheung CM, Chee SP. Jarisch-Herxheimer reaction: paradoxical worsening of tuberculosis chorioretinitis following initiation of antituberculous therapy. Eye 2009; 23: 1472-1473.

9 McAllister WA, Thompson PJ, Al-Habet SM, Rogers HJ Rifampicin reduces effectiveness and bioavailability of prednisolone. Br Med J (Clin Res Ed) 1983; 286: 923-925. 